\title{
AVALIAÇÃO CLÍNICA DA SENSIBILIDADE EM INDIVÍDUOS COM DIABETES MELITO
}

Ana Claudia de Souza Fortaleza ${ }^{1}$, Alessandra Rezende Martinelli ${ }^{1}$, Andrea Jeanne Lourenço Nozabieli ${ }^{1}$, Alessandra Madia Mantovani ${ }^{2}$, Marcela Regina de Camargo ${ }^{3}$, Cristina Elena Prado Teles Fregonesi ${ }^{4}$, Eliane Ferrari Chagas ${ }^{4}$, Dalva Minonroze Albuquerque Ferreira ${ }^{4}$, Claudia Regina Sgobbi de Faria ${ }^{4}$

${ }^{1}$ Discente de Mestrado em Fisioterapia, ${ }^{2}$ Discente de graduação em Fisioterapia da FCT/UNESP; ${ }^{3}$ Fisioterapeuta, mestre em Fisioterapia pela FCT/UNESP; ${ }^{4}$ Docente do departamento de Fisioterapia da FCT/UNESP. anafortalezaunesp@yahoo.com.br Agência financiadora: CAPES

\section{RESUMO}

Este estudo teve como objetivo realizar uma avaliação clínica de fácil aplicabilidade e baixo custo para detectar a presença da neuropatia diabética periférica (NDP) em indivíduos com DM, bem como correlacionar a pontuação obtida com o teste de estesiometria e o MNSI. Foram avaliados 44 participantes, com diagnóstico de DM. A NDP foi diagnosticada pelo Michigan Neuropathy Screening Instrument (MNSI) e por estesiometria com monofilamentos Semmes-Weinstein. Para a análise estatística foi realizada a análise descritiva e o Teste de Correlação linear de Spearman para a correlação entre o último monofilamento que o indivíduo sentiu no teste do estesiômetro e o escore obtido no MNSI. 18 (40,9\%) indivíduos apresentaram diagnóstico de neuropatia pelo MNSI e $30(68,2 \%)$ puderam ser diagnosticados como neuropatas pela estesiometria, os dois testes apresentaram uma correlação significante, sendo então, possível concluir que o que esses dois testes são efetivos quando utilizados em conjunto para tal diagnóstico.

Palavras-chave: diabetes mellitus, neuropatias diabéticas, avaliação, sensação.

\section{CLINICAL ASSESSMENT IN SUBJECTS WITH DIABETES MELLITUS}

\begin{abstract}
This study aimed to perform a clinical evaluation of low cost and easily applied to detect the presence of peripheral diabetic neuropathy (DPN) in individuals with DM and to correlate the score of the esthesiometry's test and MNSI. We evaluated 44 participants with a diagnosis of DM.The NDP was diagnosed by the Michigan Neuropathy Screening Instrument (MNSI) and by esthesiometry with Semmes-Weinstein monofilaments. Eighteen (40.9\%) subjects were diagnosed with neuropathy by MNSI and $30(68.2 \%)$ by esthesiometry, the two tests showed a significant correlation, then, is possible to conclude that these two tests are effective when used together for such a diagnosis.
\end{abstract}

Keywords: diabetes mellitus, diabetic neuropathies, evaluation, sensation. 


\section{INTRODUÇÃO}

O Diabetes Mellitus (DM) pode ser compreendido como uma síndrome clínica na qual ocorrem distúrbios do metabolismo de carboidratos, lipídios e proteínas, decorrente da falta absoluta ou relativa de insulina ou deficiência na sua ação ${ }^{(1)}$. Trata-se de uma doença de importância mundial que vem se tornando um problema de Saúde Pública, devido ao número de pessoas afetadas, às complicações e incapacitações trazidas pela doença e ao elevado custo financeiro da sua abordagem terapêutica ${ }^{(2)}$.

A neuropatia diabética periférica (NDP) é

uma das complicações mais comuns na progressão do $\mathrm{DM}^{(3)}$ e leva a efeitos prejudiciais nas habilidades físicas, emocionais e nas funções sociais dos pacientes. Muitos experimentam dor ou desconforto, limitação nas atividades, ansiedade, depressão e diminuição na produtividade do trabalho(4).

Essa complicação é inicialmente sensitiva, com déficits na sensibilidade tátil, vibratória e proprioceptiva, no entanto, cursa com uma degeneração progressiva dos nervos periféricos que atingem principalmente os membros inferiores, podendo causar déficits motores, com alterações no trofismo muscular, especialmente nos músculos intrínsecos dos pés e tornozelos ${ }^{(5)}$.

As alterações neurológicas da neuropatia juntamente com as alterações vasculares podem causar mais uma complicação comum nos diabéticos crônicos: o pé diabético, caracterizado pela presença de lesões nos pés, em decorrência das alterações vasculares e neurológicas peculiares do $\mathrm{DM}^{(6)}$. A síndrome do pé diabético é definida como quaisquer lesões, agudas ou crônicas, que ocorrem no pé dos pacientes diabéticos, com infecção, ulceração e/ou destruição de tecidos profundos ${ }^{(7)}$.

À medida que a neuropatia progride desenvolvem-se pontos de alta pressão, que podem ser identificados por áreas com calosidades que originam úlceras e focos de infecção devido à pressão continuada e à abrasão $^{(8)}$. Essas lesões nos pés podem desencadear uma das complicações mais temidas, a amputação. No entanto, essa é uma situação que pode ser evitada mediante a detecção precoce dos pés em risco ${ }^{(2)}$.

Muitos autores têm enfatizado a importância de um trabalho preventivo em indivíduos diabéticos, para que sejam orientados quanto aos cuidados gerais para prevenção de incapacidades e, assim, reduzir a possibilidade de desenvolver problemas futuros ${ }^{(9,10)}$. Há um consenso de que o pé diabético seja uma complicação passível de prevenção a partir da atuação de um grupo multidisciplinar ${ }^{(11)}$, com ações educativas como a conscientização da população diabética, de médicos e profissionais da saúde, juntamente com diagnóstico precoce de alterações neuro-sensório-motoras.

\section{OBJETIVO}

Realizar uma avaliação clínica de fácil aplicabilidade e baixo custo para detectar a presença da neuropatia diabética periférica (NDP) em indivíduos com DM, bem como correlacionar o resultado obtido no teste de estesiometria e a pontuação do MNSI.

\section{METODOLOGIA}

Esta pesquisa consistiu em um estudo transversal onde foram avaliados 44 pacientes participantes do Projeto de Extensão Universitária "Programa Pé Diabético", com diagnóstico médico de DM. Os pacientes que aceitaram participar do estudo assinaram o Termo de Consentimento Livre e Esclarecido.

Os sujeitos foram submetidos a uma avaliação inicial, com dados pessoais, aspectos relacionados à diabetes (tipo de diabetes) e teste de glicemia pós-prandial. 
Em seguida, os pacientes foram avaliados quanto à presença ou não de Neuropatia Diabética; para tal foi utilizado 0 Michigan Neuropathy Screening Instrument ${ }^{(12)}$, um instrumento para triagem de pacientes com alterações neuropáticas. O escore do questionário varia de zero a treze, sendo que uma pontuação igual ou superior a oito caracteriza 0 indivíduo como neuropata ${ }^{(13,14)}$.

Posteriormente, foi realizada uma avaliação somatossensitiva, por meio de estesiometria com monofilamentos SemmesWeinstein (SorriBauru®, Bauru, Brasil) que define a perda da sensação protetora no pé(15). Este é composto de seis monofilamentos de nylon que são pressionados em nove áreas plantares duas áreas dorsais, correspondentes aos dermátomos dos nervos tibial anterior e fibular comum. Durante a realização do teste 0 paciente foi orientado a permanecer em decúbito dorsal e o examinador realizou uma pressão do monofilamento sobre a pele, até que este se curvasse. Os indivíduos foram orientados a se manifestarem verbalmente todas as vezes que percebessem o toque do monofilamento em sua pele $^{(16)}$. A insensibilidade ao monofilamento de 10 $g$ é indicativa de diagnóstico de neuropatia diabética periférica ${ }^{(17)}$.

Para a análise estatística foi realizada a análise descritiva, com média e desvio padrão das variáveis e o Teste de Correlação linear de Spearman para a correlação entre o último monofilamento que o indivíduo sentiu no teste de sensibilidade e o escore obtido no MNSI. Para isso, foi necessário transformar os valores obtidos na estesiometria em uma escala numérica de 1 a 7, onde 1 correspondeu ao monofilamento de menor pressão $(0,05 \mathrm{~g})$ e 7 correspondeu à ausência total de sensibilidade somatossensitiva.

\section{RESULTADOS}

Os indivíduos avaliados apresentaram idade de 61,3 $\pm 8,55$ anos e uma glicemia de jejum

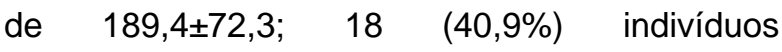
apresentaram diagnóstico de neuropatia pelo MNSI, sendo a média da pontuação obtida nesse questionário de $8,8 \pm 2,8$. Houve alteração de sensibilidade em $86,36 \%$ dos indivíduos, sendo que $30(68,2 \%)$ puderam ser diagnosticados como neuropatas por apresentarem perda da sensibilidade.

Foi realizado o Teste de Correlação de Spearman e foi visto uma correlação significante entre 0 Teste de Estesiometria pelos monofilamentos e o MNSI. Abaixo, os resultados do Teste de Correlação entre o último monofilamento que o indivíduo sentiu e o escore obtido no MNSI.

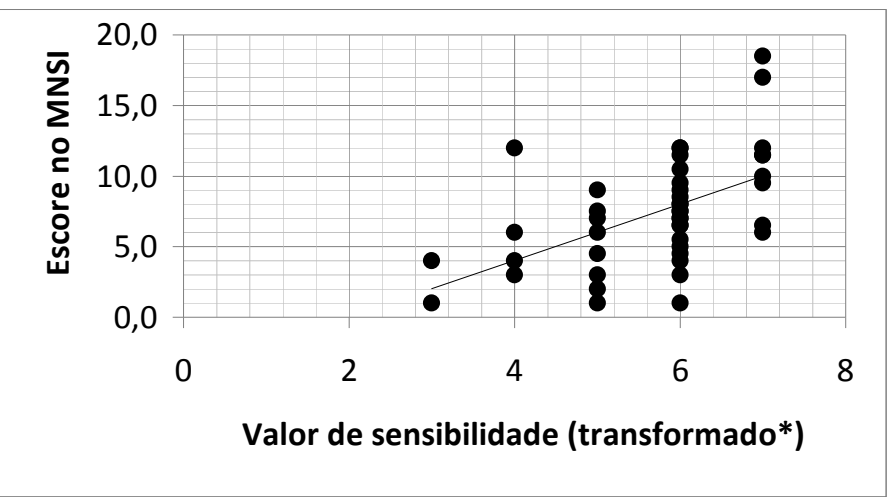

Figura 1. Teste de Correlação linear de Spearman: último monofilamento que sentiu $x$ escore no MNSI.

\section{DISCUSSÃO}

A hiperglicemia crônica, em longo prazo, leva a complicações que atingem os olhos, rins, nervos, coração e vasos sanguíneos ${ }^{(18)}$. Essas complicações, como a retinopatia, nefropatia, hipertensão arterial sistêmica, dislipidemia e neuropatias, podem causar danos à manutenção do equilíbrio dos sistemas, contribuindo para o aumento da morbidade e mortalidade dos 
pacientes diabéticos ${ }^{(19)}$, aumentando os custos dos serviços de saúde ${ }^{(20)}$.

Foi observado neste estudo que a maioria dos pacientes diabéticos avaliados apresentou alterações em relação à sensibilidade e que a média da glicemia atual foi de $189,4 \pm 72,3$, o que explica em longo prazo, as alterações sensitivas apresentadas por eles.

A perda da sensibilidade interfere muito na vida do paciente, pois diminui as aferências para o sistema de controle motor, contribuindo para disfunções como perda do equilíbrio, com alterações na marcha e na postura. Desta forma, indivíduos neuropatas apresentam maior risco a quedas, dificuldades de subir escadas, andar em ruas movimentadas, entre outras alterações ${ }^{(21)}$.

Estudo realizado por Sacco et al. ${ }^{(21)}$, avaliou a sensibilidade tátil por meio dos monofilamentos em indivíduos diabéticos, encontrando alterações em todos os indivíduos avaliados.

No presente estudo alguns indivíduos apresentaram apenas diminuição da sensibilidade (com ausência da resposta ao monofilamento de 4g) não sendo, portanto, classificado como neuropatas. No entanto, embora seja estabelecido que para o diagnóstico de neuropatia seja necessária ausência de resposta ao monofilamento de $10 \mathrm{~g}^{(17)}$, a insensibilidade ao monofilamento de $4 \mathrm{~g}$ já indica a perda da sensação protetora do pé, vulnerabilidade a lesões e perda da discriminação para quente e frio; essas alterações chamam atenção para o início da doença e podem ser identificadas precocemente para que medidas adequadas sejam tomadas, evitando assim, a progressão da doença ${ }^{(21)}$.

Moghtaderi et al. ${ }^{(13)}$, realizaram um estudo para a validação do Michigan Neuropathy Screening Instrument (MNSI) e constataram que este instrumento é de fácil aplicação e interpretação, sendo para triagem de quais pacientes deveriam ser encaminhados para estudos eletrofisiológicos. Ainda os autores sugerem que caso o paciente apresente sinais e sintomas não relatados por este instrumento, também devem ser realizados estudos eletrofisiológicos, afinal o MNSI é apenas um teste para triagem.

A utilização apenas do questionário de Michigan pode mascarar algumas alterações da neuropatia, pois as respostas dadas pelos pacientes podem não ser reais. A utilização dos monofilamentos pode comprovar as alterações de sensibilidade apresentadas pelo paciente confirmando assim, o diagnóstico.

Como observado neste estudo os dois instrumentos apresentaram um correlação significante, comprovando que esses dois testes são efetivos quando utilizados em conjunto para tal diagnóstico.

\section{CONCLUSÃO}

Foi possível observar pelo MNSI e pelo teste de estesiometria a presença de NDP na maioria dos indivíduos avaliados e que esses dois instrumentos apresentam uma correlação significante, mostrando que esses dois testes são efetivos quando utilizados em conjunto para tal diagnóstico.

Assim, uma avaliação da sensibilidade dos pés na população diabética, por meio de instrumentos de fácil aplicabilidade e baixo custo, torna-se de extrema importância para que seja realizada uma adequada orientação e prevenção, evitando maiores complicações.

\section{REFERÊNCIAS}

1. Schmid H, Neumann C, Brugnara L. O diabetes melito e a d enervação dos membros inferiores: a visão do diabetólogo. J Vasc Br. 2003; 2(1):37-48. 
2. Fajardo C. A importância do cuidado com o pé diabético: ações de prevenção e abordagem clínica. Rev Bras Med Fam e Com. 2006; 2(5).

3. Bacarin TA, Sacco ICN, Hennig EM. Plantar pressure distribution patterns during gait in diabetic neuropathy patients with a history of foot $\begin{array}{llll}\text { ulcers. Clinics 2009; } 64 \text { (2):113-120. } & \end{array}$ http://dx.doi.org/10.1590/S1807$\underline{59322009000200008}$

4. Zhao Y. Zhao Y, Ye W, Boye KS, Holcombe $\mathrm{JH}$, Hall JA, Swindle R. Prevalence of other diabetes-associated complications and comorbidities and its impact on health care charges among patients with diabetic neuropathy. Journal of Diabetes and Its Complications 2010; 24: 9-19.

http://dx.doi.org/10.1016/j.jdiacomp.2008.09.001

5. Menz HB, Menz, Lord SR, St George R, Fitzpatrick RC Walking stability and sensoriomotor function in older people with diabetic peripheral neuropathy. Arch Phys Med Reabil. 2004; 85(2):245-52.

http://dx.doi.org/10.1016/j.apmr.2003.06.015

6. Milman MHSA, Leme CBM, Borelli DT, Kater FR, Baccili ECDC, Rocha, RCM, et al. Pé Diabético: Evolução e Custo Hospitalar. Arq Bras Endocrinol Metab. 2001; 45 (5): 447-451.

7. Lopes CF. Assistência ao paciente com pé diabético. J Vasc Br. 2003; 2(1).

8. Mateus CMB. O pé diabético: uma revisão. Escola Superior de Saúde Jean Piaget/Algarve, 2005.

9. Foss NT, Foss MC. Lesões plantares do paciente diabético- pé diabético. Rev. Bras. Neurol. 1991; 26(1): 37S-38S.
10. Fregonesi CEPT, Faria CRS, Molinari SL. Avaliação sensitiva do nervo tibial através de monofilamentos em portadores de diabetes mellitus. Arq. Cienc. Saude Unipar 2002; 6(2): 111-116.

11. Chin $M H$, Cook S, Jin L, Drum, ML Harrison JF, Koppert J et al. Barriers to providing diabetes care in community health center. Diabetes Care 2001; 24: 268-74.

http://dx.doi.org/10.2337/diacare.24.2.268

12. University of Michigan Health System, Michigan Diabetes Research and Training Center (2009) MNSI - Michigan Neuropathy Screening Instrument. Available from: http://www.med.umich.edu/mdrtc/profs/survey.htm I , Acesso em: 10 July 2010.

13. Moghtaderi A, Bakhshipour A, Rashidi $\mathrm{H}$. Validation of Michigan

neuropathy screening instrument for diabetic peripheral neuropathy. Clin Neurol Neurosurg 2006; 108: 477-81. http://dx.doi.org/10.1016/j.clineuro.2005.08.003

14. Feldman EL, Stevens MJ, Thomas PK et al. A practical two-step quantitative clinical and electrophysiological assessment for the diagnosis and staging of diabetic neuropathy. Diabetes Care 1994;17:

1281-9.

\section{http://dx.doi.org/10.2337/diacare.17.11.1281}

15. Kamei N, Yamane K, Nakanishi S, Yamashita $\mathrm{Y}$, Tamura T, Ohshita $\mathrm{K}$ et al. Effectiveness of Semmes- Weinstein monofilament examination for diabetic peripheral neuropathy screening. J Diabetes Complications 2005; 19(1): 47-53. http://dx.doi.org/10.1016/j.jdiacomp.2003.12.006

16. Nather $\mathrm{A}$, Neo $\mathrm{SH}$, Chionh SB, Liew CFS, Sim EY, Chew JLL. http://dx.doi.org/10.1016/j.jdiacomp.2006.10.007A ssessment of sensory neuropathy in diabetic patients without diabetic foot problems. J Diabetes Complications 2008; 22(2): 126-131. 
17. Cavanagh PR, Simoneau GG, Ulbrecht JS. Ulceration, unsteadiness, and uncertainty - the biomechanical consequences of diabetes mellitus. J. Biomech 1993; 26 (1): 23-28. http://dx.doi.org/10.1016/0021-9290(93)90077-R

18. Laakso M. Cardiovascular disease in type 2 diabetes from population to man to mechanisms. Diabetes Care 2010; 33(2): 442-49. http://dx.doi.org/10.2337/dc09-0749

19. Cordeiro RC, Jardim JR, Perracini MR, Ramos LR. Factors associated with functional balance and mobility among elderly diabetic outpatients. Arq. Bras. Endocrinol. Metab. 2009; 53(7): 834-843. http://dx.doi.org/10.1590/S0004-

\section{$\underline{27302009000700007}$}

20. Stone MAStone MA, Wilkinson JC, Charpentier G, Clochard N, Grassi G, Lindblad U, Müller UA, Nolan J, Rutten GE, Khunti K , Evaluation and comparison of guidelines for the management of people with type 2 diabetes from eight European countries. Diabetes Research and Clinical Practice 2010; 87: 252-260. http://dx.doi.org/10.1016/j.diabres.2009.10.020

21. Sacco ICN Sacco ICN, Sartor CD, Gomes AA, João SMA, Cronfli, R. Avaliação das perdas sensório-motoras do pé e tornozelo decorrentes da neuropatia diabética. Rev Bras Fisioter 2007; 11(1):27-33. 